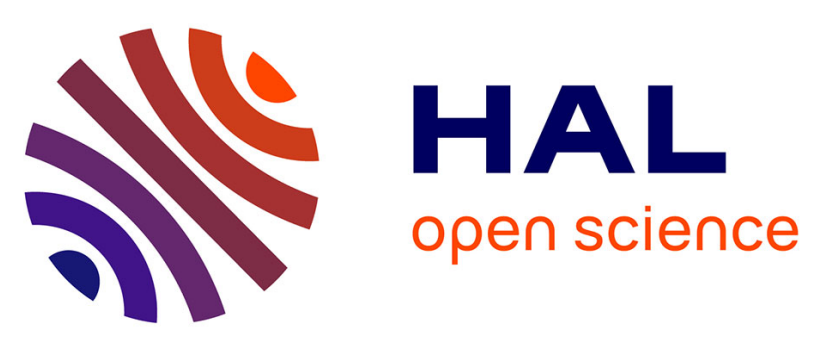

\title{
Theoretical and Experimental Study of the Phase Noise of Opto-Electronic Oscillators based on High Quality Factor Optical Resonators
}

Aude Bouchier, Khaldoun Saleh, Pierre-Henri Merrer, Olivier Llopis, Gilles Cibiel

\section{To cite this version:}

Aude Bouchier, Khaldoun Saleh, Pierre-Henri Merrer, Olivier Llopis, Gilles Cibiel. Theoretical and Experimental Study of the Phase Noise of Opto-Electronic Oscillators based on High Quality Factor Optical Resonators. IEEE International Frequency Control Symposium, Jun 2010, Newport Beach, United States. 5p. hal-00496888

\section{HAL Id: hal-00496888 https://hal.science/hal-00496888}

Submitted on 1 Jul 2010

HAL is a multi-disciplinary open access archive for the deposit and dissemination of scientific research documents, whether they are published or not. The documents may come from teaching and research institutions in France or abroad, or from public or private research centers.
L'archive ouverte pluridisciplinaire HAL, est destinée au dépôt et à la diffusion de documents scientifiques de niveau recherche, publiés ou non, émanant des établissements d'enseignement et de recherche français ou étrangers, des laboratoires publics ou privés. 


\section{Theoretical and Experimental Study of the Phase Noise of Opto-Electronic Oscillators based on High Quality Factor Optical Resonators}

\author{
A. Bouchier, K. Saleh, P.-H. Merrer, O. Llopis \\ LAAS-CNRS \\ Université de Toulouse ; UPS, INSA, INP, ISAE ; LAAS \\ Toulouse, France \\ bouchier@laas.fr
}

\author{
G. Cibiel \\ CNES \\ Toulouse, France
}

\begin{abstract}
In order to improve the phase noise of high purity OEO based on optical resonators, like resonant fibre rings, these oscillators have been theoretically studied in terms of white frequency noise. We present here the optimization of the performances of a fibre ring resonator and the experimental phase noise results we obtained for the OEO based on an optimized very high quality factor optical resonator.
\end{abstract}

\section{INTRODUCTION}

The design of new compact and high performances oscillators is linked to the investigation of new resonators. A disadvantage of classical microwave (electromagnetic) resonances is that their quality factor $(\mathrm{Q})$ decreases when the frequency increases, limiting their efficiency to build high purity sources at very high frequencies (millimetre wave range). The best resonators performances in terms of quality factor are today obtained using whispering gallery modes in sapphire crystal cylindrical resonators, with an unloaded quality factor close to $210^{5}$ around $10 \mathrm{GHz}$. An increase of this quality factor is possible with the use of cryogenic sapphire resonators, leading to unloaded quality factors higher than $10^{9}$ around $10 \mathrm{GHz}$ [1]. However, this solution is bulky and difficult to implement in practical applications.

An elegant method to reduce the oscillators' dimensions and/or to increase the Q factor is to carry the microwaves on another wave, like acoustic or optical waves [2] for example. In this study, an optical carrier at $1.55 \mu \mathrm{m}$ is used. This carrier is a laser developed for telecommunication applications. Thanks to this optical carrier, the microwave resonator becomes an optical resonator, with higher Q and relatively low dimensions due to the shorter wavelengths. It is thus possible to design new devices featuring better performance than classical microwave devices.

The optical resonator generates an optical frequency comb with microwave spacing. This spacing, the free spectral range (FSR), is directly related to the resonator dimensions. Such an optical resonator features a $3 \mathrm{~dB}$ bandwidth which is maintained when it is used to filter microwaves. Thus, to the optical $\mathrm{Q}_{\mathrm{opt}}$ factor corresponds an equivalent RF quality factor $\mathrm{Q}_{\mathrm{RF}}$. This quality factor is the product of $\mathrm{Q}_{\mathrm{opt}}$ and the RF to optical frequencies ratio (1).

$$
Q_{R F}=Q_{\text {opt }} \cdot \frac{f_{R F}}{f_{\text {opt }}}
$$

Thanks to this relation, it is clear that the RF equivalent quality factor increases with the RF frequency for a fixed optical frequency. To reach quality factors as high as those obtained with microwave resonators, $Q_{\text {opt }}$ should be higher than $10^{9}$ because of the frequency ratio, which is as high as $10^{4}$ for a carrier at $1.55 \mu \mathrm{m}$ (around $194 \mathrm{THz}$ ) and a microwave signal around $20 \mathrm{GHz}$. With this challenging optical Q factor, these optical resonators can lead to an elegant alternative to microwave resonators and also to more compact devices if the optical parts can be integrated or assembled in a small volume.

The use of an optical carrier in microwave photonics domain is in fact a classical solution in the case of the optical delay lines. These lines are few-km-long optical fibres, with equivalent RF quality factors that can reach $10^{6}$ at $10 \mathrm{GHz}$. They have been used for example to stabilize oscillators [3]. Although they are easy to use and actually well-known, they remain bulky devices and there thermal stabilization is difficult.

In the case of optical resonators, a first solution is similar to the high Q RF resonators: the use of optical WGM resonances $[4,5]$. These devices are spheres, torus or disks, in which the optical wave circulates on the whispering gallery modes. These structures have to be transparent at $1.55 \mu \mathrm{m}$, and are constituted generally from silica, quartz, calcium $\left(\mathrm{CaF}_{2}\right)$ or magnesium fluoride $\left(\mathrm{MgF}_{2}\right)$ and so on... These resonators present really high optical quality factors with 
demonstrated values from $10^{8}$ to $10^{11}$ at $1.55 \mu \mathrm{m}[4,5]$, but their use in a system is delicate, particularly the coupling of the optical carrier is really difficult with the use of tapered fibres or prisms, for example.

A last approach is the use of the ability of fibered optics, which has been developed for telecommunications around $1.55 \mu \mathrm{m}$, and we present here the principle and use of fibre ring resonators. To predict their efficiency in terms of quality factors and phase noise when they are included in an optoelectronic oscillator (OEO) scheme, a simulation tool is presented here. The results are compared with experimental data, which have been fully described in [6]. The optimization approach of the white frequency noise contribution we propose is an extension to the OEO case of a classical method used for microwave oscillators [7]. The experimental results obtained with a new optimized resonator are also presented.

\section{RESONANT FIBRE RINGS}

Optical telecommunications leaded to the development of optical fibres, pigtailed lasers emitting around $1.55 \mu \mathrm{m}$, and passive or active optoelectronic components. They allow us to build easily at this wavelength an optical resonator using two fibered couplers linked with single-mode fibres at $1.55 \mu \mathrm{m}$ like Corning SMF28 fibres (Fig. 1). This resonator will thus generate a transverse single frequency comb. Thanks to the compatibility with pigtailed lasers, the coupling of the optical carrier in the optical resonator is stable and the system is potentially integrated in a compact device.

The ring we study here has a total length of $10 \mathrm{~m}$ and is constituted by two optical couplers with coupling ratios $\mathrm{T} / \kappa$ close to $90 / 10 \%$ (Fig. 1). The connection between the fibres is made through splices. This ring generates a frequency comb with a FSR of $20 \mathrm{MHz}$.

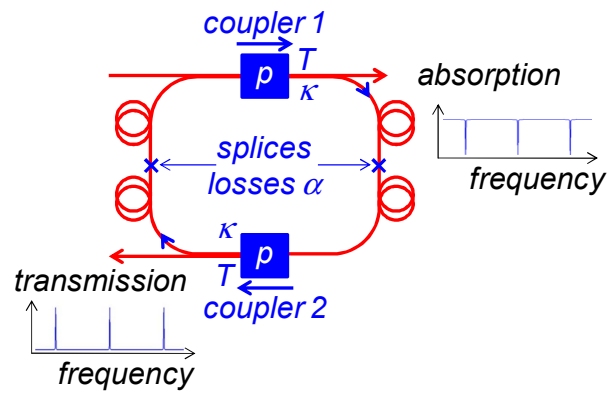

Figure 1. Scheme of the fibre ring resonator and resonant modes in transmission and absorption.

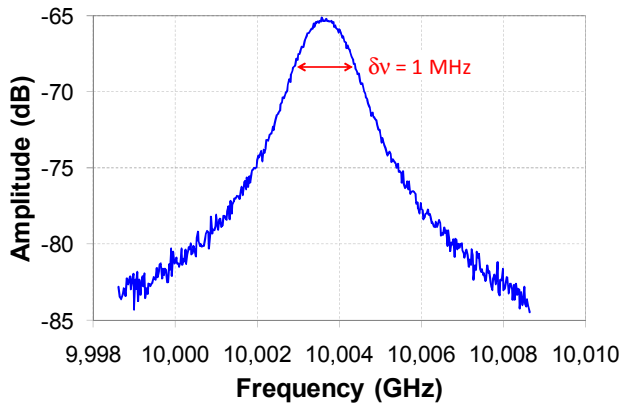

Figure 2. Resonance of the stabilized 10m-long fibre ring.
The characterization of the fibre ring thanks to the stabilization of the laser on a ring resonance [6] leads to the determination of a quality factor of $2 \times 10^{8}$ at $1.55 \mu \mathrm{m}$ (Fig. 2).

\section{OPTOELECTRONIC OSCILLATORS}

The OEO we have built thanks to this fibre ring is schematically described on Fig. 3 and has been fully described in [6].

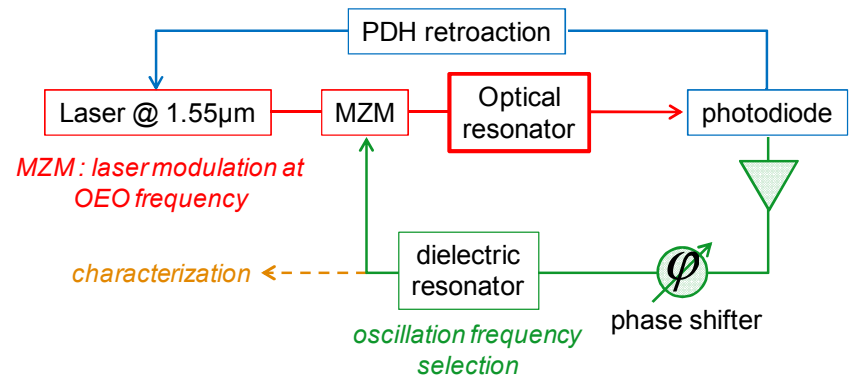

Figure 3. Principle of the optoelectronic oscillator at $10 \mathrm{GHz}$.

The phase noise spectrum of this OEO and its spectrum have been measured with an Agilent E5052B signal analyzer (Fig. 4).

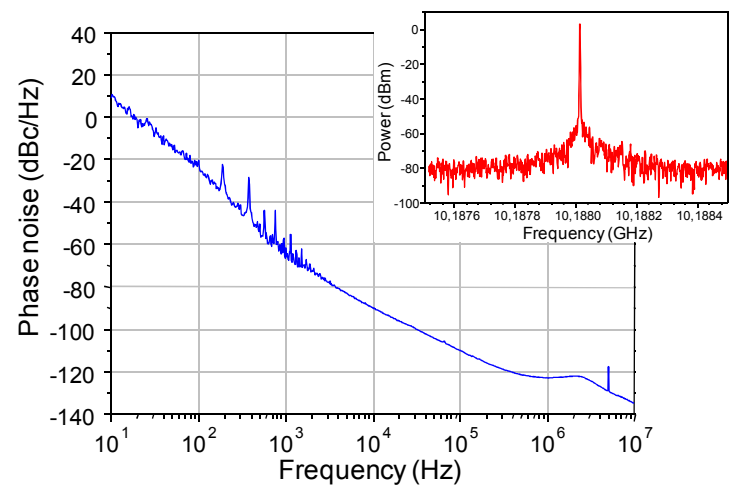

Figure 4. Phase noise measurement and spectrum (in inset) for the optoelectronic oscillator at $10 \mathrm{GHz}$.

This phase noise spectrum presents four different areas. In the first one, between $10 \mathrm{~Hz}$ and $1 \mathrm{kHz}$, the noise slope of $-30 \mathrm{~dB} / \mathrm{dec}$ corresponds to an important $1 / \mathrm{f}$ frequency noise contribution. The spurious peaks that are visible between 0.1 and $1 \mathrm{kHz}$ can be due to thermal or mechanical fluctuations of the fibre ring resonator or another noise linked to the optical amplifier. The white frequency noise contribution dominates the spectrum between 1 and $400 \mathrm{kHz}$, as we can see it through the $-20 \mathrm{~dB} / \mathrm{dec}$ slope of the phase noise. The phase noise floor appears after $400 \mathrm{kHz}$ with a value of about $-120 \mathrm{dBc} / \mathrm{Hz}$. The final decrease of the noise above $3 \mathrm{MHz}$ is linked to the filter effect of the dielectric resonator.

The knee frequency $f_{c}$ at the frontier between the white frequency noise and the phase noise floor is linked to the loaded microwave quality factor $\left(\mathrm{Q}_{\mathrm{RF}}\right)$ by the relation (2)

$$
f_{c}=\frac{f_{R F}}{2 Q_{R F}}
$$


with $f_{R F}$ the microwave frequency [8]. With a frequency $f_{c}$ at this limit of $400 \mathrm{kHz}$, the loaded microwave quality factor is $1.210^{4}$ at $10 \mathrm{GHz}$, which is in good agreement with an optical quality factor of $210^{8}$ at $1.55 \mu \mathrm{m}$ for the resonator.

In spite of the relatively good loaded quality factor of the fibre ring, the OEO presents a high phase noise level. Effectively, the carrier to noise ratio due to this resonator is not optimized and the transmission losses of the ring are important. These losses are mainly due to the coupling ratio of its couplers. They directly influence the white frequency noise of the oscillator.

The phase noise induced by the optical and electrical amplifiers can have an effect on the $1 / \mathrm{f}$ noise. We have thus studied their influence thanks to residual phase noise measurements. The measured residual phase noise can then be introduced in a simplified model of the OEO, following Leeson's approach [8]. Taking into account the OEO Q factor, we can see that their contribution to the OEO phase noise is not high enough to really influence the oscillator behaviour, with a level about 15 to $30 \mathrm{~dB}$ lower than the measured OEO phase noise (Fig. 5).

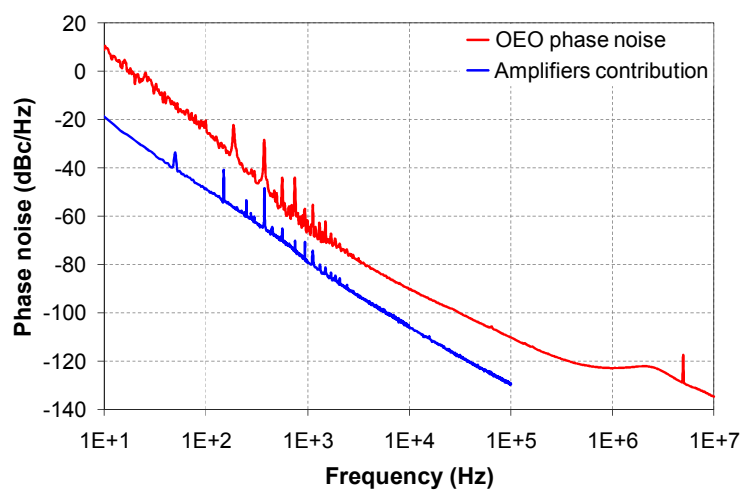

Figure 5. Contribution of the amplifiers phase noise to the OEO noise and comparison with the measured OEO spectrum at $10 \mathrm{GHz}$.

The excess noise may be induced by the laser stabilization loop, the laser noise and/or an optical resonator that are not completely optimized and may present some thermal, mechanical and electronic instabilities at low frequencies. However, for the white frequency noise part of the spectrum, the noise contributions can be more easily calculated as they are related to the optical carrier to the noise ratio. A theoretical study of the contribution of the white frequency noise of the optical resonator has thus been performed.

\section{White FREQUENCY NOISE SIMULATION OF THE OEO}

The theoretical study of the OEO occurs in two steps : we have first to model the resonator itself and then its influence on the oscillator phase noise.

\section{A. Fibre Ring Resonator modeling}

The fibre ring is modelled in terms of electrical fields [9]. With this model, we can predict the FSR, the mode width and thus the optical quality factor of the resonator.

The ring is described through its length, the characteristics of the couplers in terms of transmission $(\mathrm{T})$, coupling $(\kappa)$ and losses (p) coefficients. We also take into account the intrinsic losses $(\alpha)$ of the ring such as splicing losses (Fig. 1).

\section{B. White Frequency Noise modeling}

The OEO white frequency noise contribution, resulting from the optical carrier to noise ratio is then evaluated. Inside the OEO loop bandwidth, it depends on the offset frequency $f_{m}$, the optical quality factor $Q_{o p t}$ and the noise to carrier ratio (NCR) as shown in relation (3).

$$
L\left(f_{m}\right)=20 \cdot \log \left(\frac{f_{o p t}}{2 \cdot \sqrt{2} \cdot Q_{o p t} \cdot f_{m}}\right)+10 \cdot \log (N C R)
$$

The NCR (4) depends on the measured optical power $\mathrm{P}_{\mathrm{m}}$, the sensitivity of the photodetector $\sigma$, the modulation ratio $\mathrm{m}$, and on the different noise contributions:

$$
N C R=\frac{2\left(S_{R I N}+S_{t h}+S_{\text {shot }}\right)}{P_{m}^{2} \cdot \sigma^{2} \cdot m^{2}}
$$

with the thermal noise $S_{\text {th }}(5)$, including the temperature $T$, the Boltzman constant $\mathrm{k}$, and the photodiode load resistance $\mathrm{R}$

$$
S_{t h}=\frac{4 \cdot k \cdot T}{R}
$$

the shot noise $S_{\text {shot }}(6)$, with the elementary charge $q$

$$
S_{\text {shot }}=2 \cdot q \cdot \sigma \cdot P_{m}
$$

the laser noise $\mathrm{S}_{\mathrm{RIN}}$ (7), taking into account the relative intensity noise (RIN) of the laser.

$$
S_{R I N}=P_{m}^{2} \cdot \sigma^{2} \cdot R I N
$$

\section{Comparison with the experimental phase noise}

With this model, we can completely determine the couplers characteristics and the ring losses. In this aim, we compare the experimental measurement of the OEO phase noise to the simulated result. By adjusting the couplers coefficients and losses and the ring losses, we can fit the experimental data. The uniqueness of the solution is guaranteed when we add to this fit the comparison between the theoretical $\mathrm{Q}_{\mathrm{opt}}$ and the experimental value.

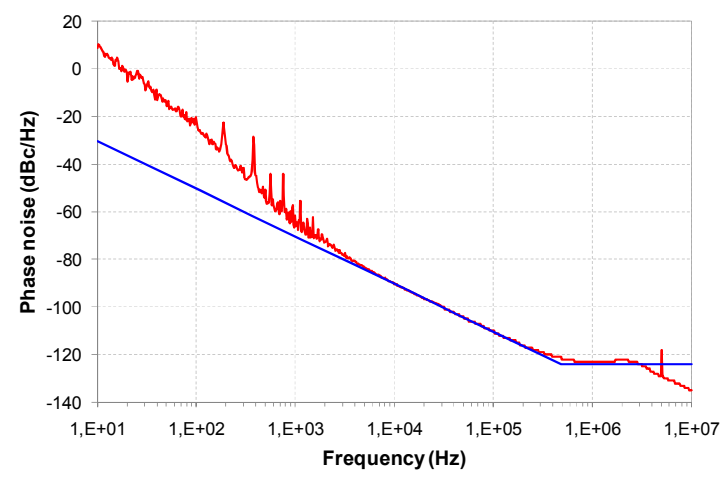

Figure 6. Simulated phase noise spectrum (in blue) for the white frequency noise contribution area (1 to $400 \mathrm{kHz}$ ) and experimental results (in red). 
The comparison between the experimental and the theoretical data is shown in Fig. 6. The model of white frequency noise is valid only from 1 to $400 \mathrm{kHz}\left(\mathrm{f}_{\mathrm{c}}\right)$, frequency range on which the phase noise is dominated by the white frequency noise contribution. With a good agreement between the model and these data (phase noise spectrum and $\mathrm{Q}_{\text {opt }}$ value), we can determine that the transmission $\mathrm{T}$ of the couplers is $0.86 \pm 0.04$, corresponding to a coupler ratio $\mathrm{T} / \kappa$ of $86 / 14 \%$ with couplers losses $\mathrm{p}$ around $0.59 \mathrm{~dB}$. The splices losses $\alpha$ are determined to be close to $0.005 \mathrm{~dB}$.

\section{OPTIMIZATION OF THE FIBRE RING RESONATOR}

The $\mathrm{Q}_{\text {opt }}$ factor of the fibre ring and its contribution to the OEO phase noise are closely linked to the fibre length, the characteristics of the couplers and the losses in the ring. The decrease of the fibre ring losses and of the coupling factors of the couplers increases $Q_{\text {opt }}$. However the decrease of the coupling factors degrades also the carrier to noise ratio. We thus should find a trade-off between low coupling coefficients, and thus high $\mathrm{Q}_{\text {opt }}$, and high NCR to obtain the best results in terms of OEO phase noise (3). For this analysis of the influence of the ring parameters, we suppose that the both couplers are identical.

\section{A. Influence of the fibre ring length}

The fibre ring length has a direct influence on the resonator quality factor. Effectively the quality factor increases with the fibre length. For example, a fibre ring resonator built on ideal 99/1\% couplers presents a $Q_{\text {opt }}$ of $2 \times 10^{8}$ at $1.55 \mu \mathrm{m}$ for a length of $1 \mathrm{~m}$, and a $Q_{\text {opt }}$ of $5 \times 10^{9}$ for a length of $20 \mathrm{~m}$. In our case, we limit the total ring length at $20 \mathrm{~m}$. This limitation is due to our OEO experimental set-up that includes a dielectric filter with a full-width at halfmaximum of $3 \mathrm{MHz}$, which aim is to select one RF resonance. To guarantee a single frequency behaviour of the OEO, the side mode suppression ratio has to be high enough and the resonator FSR higher than $10 \mathrm{MHz}$. We will thus investigate on a $20 \mathrm{~m}$-long fibre ring resonator for the study of the influence of the other parameters.

\section{B. Influence of the coupling factor and losses of the couplers}

To study the influence of the couplers losses on the choice of coupler transmission, we consider that the intrinsic losses of the ring are fixed to the splices losses value $(0.02 \mathrm{~dB})$. Fig. 7 shows the presence of an optimum value of coupler transmission for each value of couplers losses.

For example, a couplers losses value of $0.05 \mathrm{~dB}$ needs the use of $99.2 / 0.8 \%(21.1 \mathrm{~dB})$ couplers and for couplers losses of $0.5 \mathrm{~dB}$, we should use 94.3/1.3\% (12.5 dB) couplers.

In the same time, the quality factor of the resonator strongly decreases with the increase of the losses, from a value of $5 \times 10^{9}$ without losses to $0.1 \times 10^{9}$ for $1 \mathrm{~dB}$ losses in the case of couplers ratio of $99 / 1 \%(20 \mathrm{~dB})$.

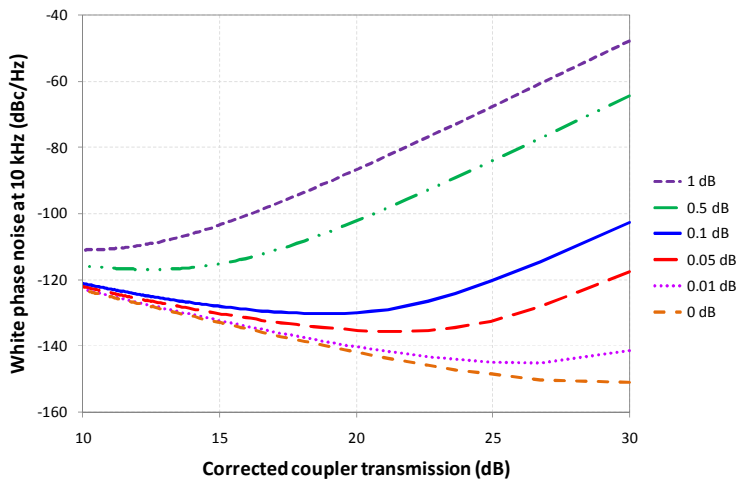

Figure 7. Influence on the coupler transmission $\mathrm{T}$ on the computed OEO phase noise for different values of couplers losses $\mathrm{p}$ from 0 to $1 \mathrm{~dB}$. The coupler transmission is corrected from the losses on this curve.

\section{Influence of the intrinsic losses of the ring}

The couplers are now considered as ideal (without losses). Fig. 8 presents the influence of splices losses, for example, on the OEO phase noise (white frequency noise only).

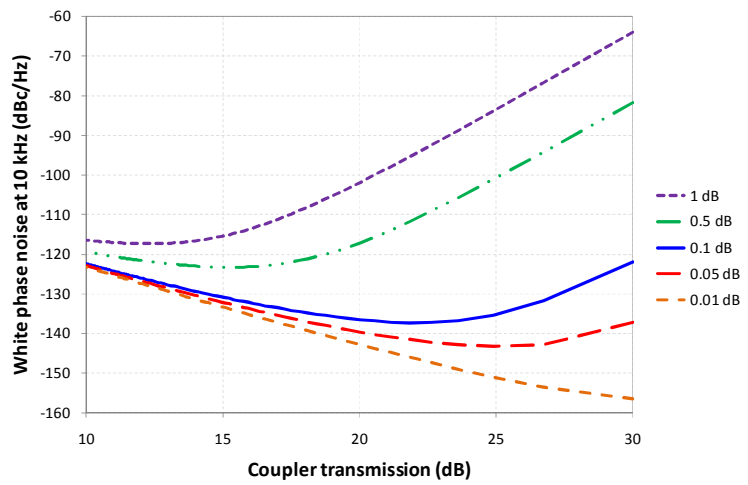

Figure 8. Influence on the coupler transmission $\mathrm{T}$ on the computed OEO phase noise for different values of intrinsic losses $\alpha$ from 0.01 to $1 \mathrm{~dB}$.

As with excess losses in the couplers, when the losses increase, the quality factor decreases and the phase noise present an optimum value in terms of couplers transmission. For losses of $0.05 \mathrm{~dB}$, the optimal coupler ratio is $99.7 / 0.3 \%$ $(24.9 \mathrm{~dB})$.

For a coupler ratio of $99 / 1 \%(20 \mathrm{~dB})$, the quality factor decreases from a value of $5.1 \times 10^{9}$ with $0.01 \mathrm{~dB}$ losses to $0.1 \times 10^{9}$ for $1 \mathrm{~dB}$ losses.

\section{Optimization of the fibre ring resonator}

With this analysis, a fibre ring constituted on two $10 \mathrm{~m}$ long fibres, with total splices losses of $0.05 \mathrm{~dB}$ and on couplers with typical losses of $0.1 \mathrm{~dB}$, the optimum of phase noise should be obtained for a ratio of $98.7 / 1.3 \%(19 \mathrm{~dB})$. In this case, we can expect an optical quality factor close to $1.6 \times 10^{9}$ at $1.55 \mu \mathrm{m}$ and a phase noise level of $-130 \mathrm{dBc} / \mathrm{Hz}$ at $10 \mathrm{kHz}$ from the carrier. We can thus notice that the best performances in terms of phase noise do not correspond to the best quality factors for the resonators. We thus have to respect a trade-off between these two parameters. 


\section{NEW OEO BASED ON AN OPTIMIZED FIBRE RING RESONATOR}

Thanks to this analysis, we have designed a new fibre ring of $20 \mathrm{~m}$, with couplers ratio of $99 / 1 \%$. The stabilization of the laser on a resonance peak of this resonator leads to a measurement of a mode width of $38 \mathrm{kHz}$ (Fig. 9), and thus to an optical quality factor of $5.1 \times 10^{9}$ at $1.55 \mu \mathrm{m}$. With this measurement, we have been able to estimate the splice losses to $0.01 \mathrm{~dB}$ and the couplers losses to $0.04 \mathrm{~dB}$. For these characteristics of the ring, the contribution of the white frequency noise is expected to be at a level of $-120 \mathrm{dBc} / \mathrm{Hz}$ at $10 \mathrm{kHz}$ from the carrier, taking in consideration that a relatively low laser power has been used in this experiment.

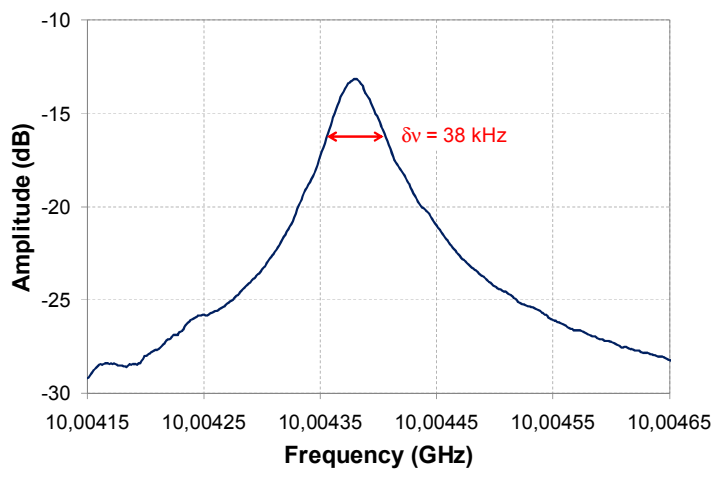

Figure 9. Resonance of the stabilized 20m-long fibre ring.

This fibre ring has been used to build an OEO at $10 \mathrm{GHz}$, which phase noise has been measured (Fig. 10).

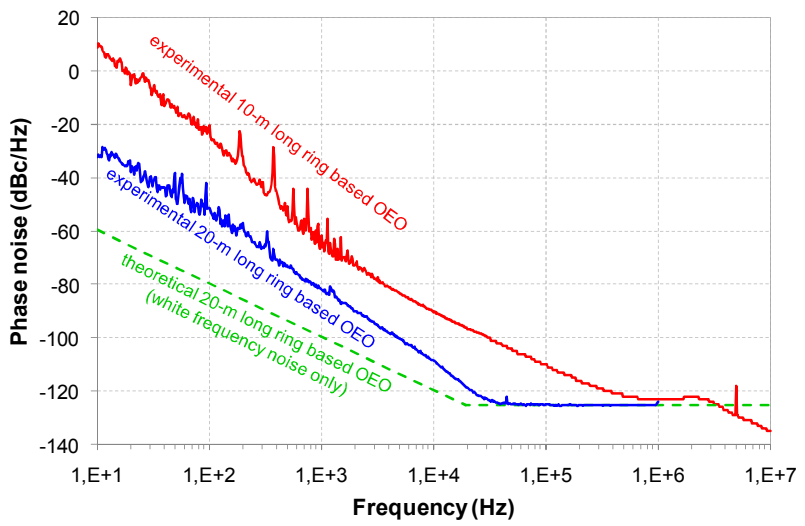

Figure 10. Phase noise measurements for the 10m-long ring based OEO (in red) and the new 20m-long ring based OEO (in blue). The green dashed curve shows the contribution of the white frequency noise of the $20 \mathrm{~m}$-long ring on the OEO phase noise.

We can observe that the phase noise level has effectively been improved with a level of $-110 \mathrm{dBc} / \mathrm{Hz}$ at $10 \mathrm{kHz}$ from the carrier, and about $-30 \mathrm{dBc} / \mathrm{Hz}$ at $10 \mathrm{~Hz}$ offset. The corner frequency confirms a RF quality factor of $2.5 \times 10^{5}$ at $10 \mathrm{GHz}$ for the OEO.

Nevertheless, the white frequency noise contribution is not predominant any more, as we can see on the comparison between the simulation and the experimental data. The 1/f noise contribution seems now to be more important in this resonator. We thus have to carefully study it to understand its origins in order to further improve this phase noise result.

\section{CONCLUSION}

Optical resonators allow the achievement of really high quality factors in the microwave frequency range, while they guarantee low dimensions. Fibered resonators, like fibre ring resonators, are easy to integrate and use. However, the OEO is a complex system in which the Q factor is not the only parameter to optimise in order to get a good phase noise performance. We have studied in this paper the white frequency noise contribution to the OEO phase noise, and we have shown that there is a trade-off to find between the resonator loaded $\mathrm{Q}$ and the optical signal to noise ratio. An important improvement of the phase noise performance has been demonstrated. However, a close to $1 / \mathrm{f}$ type noise limits now the performance of the system. Further investigations have now to be performed for a better understanding and reduction of this noise contribution.

\section{REFERENCES}

[1] A.N. Luiten, A.G. Mann and D.G. Blair, "Ultrahigh Q-factor cryogenic sapphire resonator", Electron. Letters, vol. 29 (10), pp 879-881 (1993)

[2] L. Maleki, S. Yao, Y. Ji and V. Ilchenko, "New schemes for improved opto-electronic oscillator," in International Topical Meeting on Microwave Photonics, vol. 1, pp. 177-180, 1999.

[3] X.S. Yao and L. Maleki, "Progress in the optoelectronic oscillator - a ten year anniversary review," 2004 IEEE Microwave Theory and Tech. Symp. Digest, pp. 287-290, 2004.

[4] M.L. Gorodetsky, A.A. Savchenkov and S.V. Ilchenko, "Ultimate Q of optical microsphere resonator," Opt. Letters, vol.21 (7), pp. 453-455, 1996.

[5] D.K. Armani, T.J. Kippenberg, S.M. Spillane and K.J. Vahala, "Ultrahigh-Q toroid microcavity on a chip," Nature, vol.421, pp. 925-928, 2003.

[6] P. H. Merrer, A. Bouchier, H. Brahimi, O. Llopis, and G. Cibiel, "High-Q Optical Resonators for Stabilization of High Spectral Purity Microwave Oscillators," in proc. of the 2009 IEEE EFTF-IFCS, pp. 866-869, 2009.

[7] J. Everard, "Low noise oscillators," in Microwave Symposium Digest,1992., IEEE MTT-S International, pp. 1077-1080 vol.2, 1992.

[8] D.B. Leeson, "A simple model of feedback oscillator noise spectrum," Proc. of the IEEE, vol. 54, no. 2, pp. 329-330, 1966.

[9] A. Yariv, "Critical coupling and its control in optical waveguide-ring resonator systems," IEEE Photon. Technol. Lett., vol. 14, no. 4, pp 483-485, Apr. 2002 